\title{
Self-existing objects and auto-generated information in chronology-violating space-times: A philosophical discussion
}

\author{
Gustavo E. Romero*and Diego F. Torres ${ }^{\dagger}$ \\ June 14, 2001 \\ Instituto Argentino de Radioastronomía, C.C.5, (1894) Villa Elisa, Buenos Aires, Argentina
}

\begin{abstract}
Closed time-like curves naturally appear in a variety of chronology-violating space-times. In these space-times, the Principle of Self-Consistency demands an harmony between local and global affairs that excludes grandfather-like paradoxes. However, self-existing objects trapped in CTCs are not seemingly avoided by the standard interpretation of this principle, usually constrained to a dynamical framework. In this paper we discuss whether we are committed to accept an ontology with self-existing objects if CTCs actually occur in the universe. In addition, the epistemological status of the Principle of SelfConsistency is analyzed and a discussion on the information flux through CTCs is presented.
\end{abstract}

\section{Introduction}

One of the most fascinating aspects of the general theory of relativity (GTR) is that its field equations Thave solutions where the formation of closed time-like curves (CTCs) is possible. These curves represent The world lines of any physical system in a temporally orientable space-time that, moving always in the Ifuture direction, ends arriving back at some point of its own past. Although solutions of the Einstein field Edquations (EFEs) where CTCs exist are known at least since Gödel's (1949) original work, it has been just in the last dozen of years that physicists have shown a strong and sustained interest on this topic.

The renewed attraction of CTCs and their physical implications stem from the discovery, at the end of the 1980 's, of traversable wormhole space-times (Morris et al. 1988). The subject, however, have called the 路ttention of philosophers during several decades, who have discussed paradoxes of time travel in connection with causality and logical problems (see Nahin 1999 for an extensive survey of the literature). Their treatment, unfortunately, has seldom taken into account physical considerations (see, notwithstanding, the illuminating analysis by Earman 1995a, b, among others).

In the present paper, we shall discuss two problems related with the possible existence of CTCs in GTR, which have important implications for two of the main branches of philosophy: ontology and epistemology. These problems can be formulated through the following couple of questions:

1. Are we committed, in case that CTCs actually exist in the universe, to accept an ontology with self-existing objects?

2. Can information be generated from nothing?

\footnotetext{
*Member of CONICET. E-mail: romero@irma.iar.unlp.edu.ar

${ }^{\dagger}$ E-mail: dtorres@venus.fisica.unlp.edu.ar
} 
A self-existing object is defined as any physical system that exists over a finite region of space-time but has neither starting nor ending temporal points: it is never created and it is never destroyed, but there is at least a time at which it does not exist. The first half of this paper will try to clarify the meaning of the questions mentioned above. The second half will try out some possible answers. At the end, we hope to have provided the reader with the sense that philosophical problems arisen from complex physical theories cannot be solved without appealing to the technical and conceptual tools that those same theories provide.

\section{Against CTCs: chronology protection and paradoxes}

A relativistic space-time is represented by a four-dimensional manifold $M$ equipped with a Lorentzian metric $g_{a b}$. Any space-time $\left(M, g_{a b}\right)$ with CTCs is called a chronology-violating space-time. There are two types of these space-times: those where CTCs exist everywhere (like, for instance, in Gödel space-time), and those where CTCs are confined within some regions and there exists at least one region free of them. The regions with CTCs are separated from the "well-behaved" space-time by Cauchy horizons (wormhole space-times belong to this latter type). Here we shall restrict ourselves to the second type of space-times.

The existence of CTCs and the possibility of backward time travel have been objected by several scientists championed by Hawking (1992), who proposed the so-called chronology protection conjecture: the laws of physics are such that the appearance of CTCs is never possible. The suggested mechanism to enforce chronology protection is the back-reaction of vacuum polarization fluctuations: when the renormalized energy-momentum tensor is fed back to the semi-classical EFEs, the back-reaction accumulates energy in such a way that it may distort the space-time geometry so strongly as to form a singularity, destroying the CTC at the very moment of its formation.

It has been argued, however, that quantum gravitational effects would cut the divergence off saving the CTCs (Kim and Thorne 1991). By other hand, Li et al. (1993) pointed out that the divergence of the energy-momentum tensor does not prevent the formation of a CTC but is just saying us that a full (yet unknown) quantum gravity theory must be applied. According to them, singularities, far from being physical entities that can act upon surrounding objects, are manifestations of the breakdown of the gravitational theory. In any case, we cannot draw definitive conclusions with the semi-classical tools at our disposal (see Earman 1995b for additional discussion).

But even if the energy-momentum tensor of vacuum polarization diverges at the Cauchy horizon it is not necessarily implied that CTCs must be destroyed, since the equations can be well-behaved in the region inside the horizon ( $\mathrm{Li}$ et al. 1993). In particular, wormhole space-times could be stabilized against vacuum fluctuations introducing reflecting boundaries between the wormhole mouths ( $\mathrm{Li} 1994)$ or using several wormholes to create CTCs (Thorne 1992, Visser 1997).

Recently, even Hawking has recognized that back-reaction does not necessarily enforce chronology protection (Cassidy and Hawking 1998). Although the quest for finding an effective mechanism to avoid CTCs continues, it is probable that the definitive solution to the problem should wait until a complete theory of quantum gravity can be formulated. In the meantime, the profound physical consequences of time travel in GTR should be explored in order to push this theory to its ultimate limits, to the region where the very foundations of the theory must be revisited.

A different kind of objection to CTC formation is that they allow illogical situations like the "grand- 
father" paradox ${ }_{L}^{T_{1}}$ usually interpreted as the statement that the corresponding solutions of the EFEs are "non-physical". This is a common place and has been conveniently refuted by Earman (1995a), among others (see also Nahin 1999 and references therein). Grandfather-like paradoxes do not imply illogical situations. In particular, they do not mean that local determinism does not operate in chronology-violating space-times because it is always possible to choose a neighborhood of any point of the manifold such that the equations that represent the laws of physics have appropriate solutions. Past cannot be changed (the space-time manifold is unique) but it can be causally affected from the future, according to GTR. The grandfather paradox, as pointed out by Earman 1995a, is just a way of showing us that consistency constraints must exist between the local and the global order of affairs in space-time. This leads us directly to the so-called Principle of Self-Consistency (PSC).

\section{Principle of Self-Consistency}

In space-times with CTCs, past and future are no longer globally distinct. Events on CTCs should causally influence each other along a time-loop in a self-adjusted, consistent way in order to occur in the real universe. This has been stated by Friedman et al. (1990) as a general principle of physics:

Principle of Self-Consistency: The only solutions to the laws of physics that occur locally in the real universe are those which are globally self-consistent.

When applied to the grandfather paradox, the PSC says that the grandfather cannot be killed (a local action) because in the far future this would generate an inconsistency with the global world line of the time traveler. Just consistent histories can develop in the universe. An alternative way to formulate the PSC is to state that (Earman 1995a):

The laws of physics are such that any local solution of their equations that represents a feature of the real universe must be extendible to a global solution.

The principle is not tautological or merely prescriptive, since it is clear that local observations can provide information of the global structure of the world: it is stated that there is a global-to-local order in the universe in such a way that certain local actions are ruled out by the global properties of the space-time manifold.

If the PSC is neither a tautological statement nor a methodological rule, what is then its epistemological status? It has been suggested that it could be a basic law of physics -in the same sense that the EFEs are laws of physics- (Earman 1995a). This would imply that there is some "new physics" behind the PSC. By other hand, Carlini et al. (1995) have proposed, on the basis of some simple examples, that the PSC could be a consequence of the Principle of Minimal Action. In this case, no new physics would be involved (see, however, the objections by Konstantinov (1996) on this point). Contrary to these opinions, that see in the PSC a law statement, or at least a consequence of law statements, we suggest that this principle actually is a metanomological statement, like the Principle of General Covariance among others (see Bunge 1961 for a detailed discussion of metanomological statements). This means that the reference class of the PSC is not formed by physical systems, but by laws of physical systems. The usual laws are restrictions to the state space of physical systems. Metanomological statements are laws of laws, i.e. restrictions on the global network of laws that thread the universe. The requirement of consistency constraints would then be

\footnotetext{
${ }^{1}$ The grandfather paradox: A time traveler goes to his past and kills his young grandfather then avoiding his own birth and, consequently, the time travel in which he killed his grandfather.
} 
pointing out the existence of deeper level super-laws, which enforce the harmony between local and global affairs in space-time. Just in this sense it is fair to say that "new physics" is implied.

\section{Casual loops: Self-existing objects}

\subsection{A bizarre ontology?}

Although the PSC eliminates grandfather-like paradoxes from chronology-violating space-times, other highly perplexing situations remain. The most obscure of these situations is the possibility of an ontology with self-existing objects. Let us illustrate with an example what we understand by such an object:

Suppose that, in a space-time where CTCs exist, a time traveler takes a ride on a time machine carrying a book with her. She goes back to the past, forgets the book in -what will be-her laboratory, and returns to the future. The book remains then hidden until the time traveler finds it just before starting her time trip, carrying the book with her.

It is not hard to see that the primordial origin of the book remains a mystery. Where does the book come from? This puzzle has been previously mentioned in philosophical literature by Nerlich (1981) and MacBeath (1982). Physicists, instead, have not paid much attention to it, despite the interesting fact that the described situation is apparently not excluded by the PSC: the local and global structures of the loop are perfectly harmonious and there is no casuality violations. There is just a book never created, never printed, but, somehow, existing in space-time. It has been suggested (Nerlich 1981) that if CTCs exist, then we are committed to accept an ontology of self-existing objects: they are just out there, trapped in space-time. There is no sense in asking where they are from. Even energy is conserved if we admit that the system to be considered is not only the present time-slice of the manifold but rather the two slices connected by the time loop: the energy removed from the present time $\left(M_{\mathrm{book}} c^{2}\right)$ is deposited in the past.

It is our view, however, that the acceptance of such a bizarre ontology proceeds from an incorrect application of the PSC. This principle is always discussed within the context of GTR, although actually it should encompass all physical laws. A fully correct formulation of the PSC should say laws of nature where in the formulation given above it is said laws of physics. What should be demanded is total consistency and not only consistency in the solutions of the EFEs. In particular, when thermodynamics is included in the analysis, the loop of a self-existing objects becomes inconsistent because, due to entropic degradation, the final and initial states of the object do not match. Moreover, even more strange paradoxes, related to human self-reproduction like the amazing Jocasta paradox (Harrison 1979), can be proven to be nonconsistent when the laws of genetics are taken into account.

We shall see that any time loop, described by any physical system more complex than an ideal fluid in a general wormhole space-time, undergoes entropy changes that make the loop inconsistent, and the universe free from self-existing objects.

The projection of the derivative of the stress-energy tensor onto the four-velocity gives,

$$
u^{i} \frac{\partial T_{i}^{k}}{\partial x^{k}}=u_{i} \frac{\partial \tau_{i}^{k}}{\partial x^{k}}-T \frac{\partial\left(\sigma u^{i}\right)}{\partial x^{i}}+\mu \frac{\partial \nu^{i}}{\partial x^{i}}=0
$$

where $\mu=(w-T \sigma) / n$ is called chemical potential. This equation can be re-written as the total divergence 
of a new entropy flux (see Landau and Lifchitz 1975 for details),

$$
\frac{\partial \sigma^{i}}{\partial x^{i}}=\frac{\partial}{\partial x^{i}}\left(\sigma u^{i}-\frac{\mu}{T} \nu^{i}\right)=-\nu^{i} \frac{\partial}{\partial x^{i}}\left(\frac{\mu}{T}\right)-\frac{\tau_{i}^{k}}{T} \frac{\partial u^{i}}{\partial x^{k}}
$$

The explicit forms of $\tau_{i k}$ and $\nu_{i}$ are given by

$$
\begin{gathered}
\tau_{i k}=-\eta\left(\frac{\partial u_{i}}{\partial x^{k}}+\frac{\partial u_{k}}{\partial x^{i}}+u_{k} u^{l} \frac{\partial u_{i}}{\partial x^{l}}+u_{i} u^{l} \frac{\partial u_{k}}{\partial x^{l}}\right)-\left(\zeta-\frac{2}{3} \eta\right) \frac{\partial u^{l}}{\partial x^{l}}\left(g_{i k}+u_{i} u_{k}\right), \\
\nu_{i}=-\frac{\kappa}{c}\left(\frac{n T}{w}\right)^{2}\left[\frac{\partial}{\partial x^{i}}\left(\frac{\mu}{T}\right)+u_{i} u^{k} \frac{\partial}{\partial x^{k}}\left(\frac{\mu}{T}\right)\right] .
\end{gathered}
$$

These two tensors, where $\eta$ and $\zeta$ are viscosity coefficients and $\kappa$ is the conductivity, make $\sigma^{i}$ a monotonically increasing magnitude, in agreement with the Second Law of Thermodynamics. The above equations are valid for systems which are not affected by the gravitational field. In order to study more general situations, we change the four velocity to $u^{0}=1 / \sqrt{-g_{00}}$ and $u^{\alpha}=0$, a system in free fall in the field, and we also change ordinary derivatives to covariant ones $(, \rightarrow ;)$.

It is clear that any real fluid will increase its entropy while traveling along its world line (Landau and Lifchitz 1975, Misner et al. 1973). There remains the possibility, however, that particular non-flat spacetimes have a metric such that a closed trajectory results in a zero net entropy change due to cancellation of the two terms in the right member of Eq. (i2i). We will now consider trajectories in general wormhole space-times in order to explore what happens with CTCs where real physical systems could be trapped.

Many of the analytical wormhole solutions that were presented in the literature, both for general relativity and other gravity theories (e.g. Hochberg and Visser 1997; Anchordoqui et al. 1997, 1998), are given in a static, spherically symmetric form, where the metric is constrained to yield the following interval,

$$
d s^{2}=-e^{2 \alpha} d t^{2}+e^{2 \beta} d r^{2}+r^{2} d \Omega^{2} .
$$

Here, $d \Omega^{2}=d \theta^{2}+\sin ^{2} \theta d \phi^{2}$ leads to the angular part of $g_{i k}$. Both $\alpha$ and $\beta$ are functions (associated with the metric potentials) only dependent on $r$ because of the spherical symmetry assumed. These functions have to fulfill standard wormhole space-time conditions such us the flaring out of the throat. The specific form of these conditions are unnecessary for our current purpose. Using the metric ( $\left(\begin{array}{l}1 \\ 2\end{array}\right)$ one can immediately prove that

$$
\sigma_{; i}^{i}=\frac{1}{\sqrt{-g}}\left(\sqrt{-g} \sigma^{i}\right)_{, i}=\sigma_{, i}^{i}+\sigma^{i} \frac{1}{\sqrt{-g}}(\sqrt{-g})_{, i}=\frac{\kappa}{c}\left(\frac{n T}{w}\right)^{2} \sum_{i=1}^{3}\left(\frac{\mu}{T}\right)_{, i}^{2} .
$$

Here, sub-indices from 1 to 3 stands for derivatives with respect to $r, \theta$ and $\phi$. This equation represents the increase in the entropy flux when a non-ideal fluid is moving in the gravitational field of equation ( $\left(\bar{b}_{1}^{-1}\right)$. Any physical time loop will have non-null length and consequently for the object in the loop we shall have $\sigma_{; i}^{i}>0$.

Note that, due to the form of the metric, all factors of $\sigma_{; i}^{i}$ involving viscosity coefficients cancel out

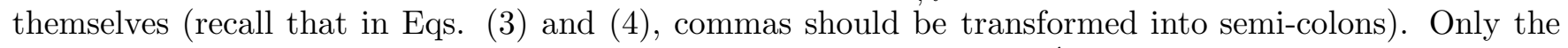
conductivity appears in the entropy flux divergence. Note also that $\sigma_{; i}^{i}$ is positive definite, disregarding the form of the functions $\alpha$ and $\beta$. There is no way in which a real fluid, with non-zero conductivity, may traverse the space-time given in Eq. $\left(\underline{5}_{1}^{\prime}\right)$ without increasing its entropy. Any object using a wormhole as 
a time machine will be degraded in a such a way that a CTC can not be formed according to the PSC. Self-existing objects are, consequently, physically impossible constructs in wormhole space-times .

\section{Casual loops: Auto-generated information}

\subsection{Information coming from nothing?}

Let us now consider again the time traveler mentioned before, and suppose that when she arrives to the past she looks for the author of the book she has brought with her. Imagine that once the author is found, she gives the book to him before he actually wrote it, and then comes back to the future. The book stays in time traveler's past and naturally ages as time goes by. The yet-to-be author reads and likes the book, and decides that the material deserves wide publicity. So, he types the text and sends the manuscript to the editor signed with his name. The editor agrees with the publication and the book is edited. Some years latter the time traveler takes one copy and carries it to the past, where she gives it to the author. Where does the information contained in the book come from?. Here, there is no problem with physical time loops, since the object (the book) normally ages with time. The PSC seems not to be violated in any respect.

This kind of casual loops are called information paradoxes (see, for instance, Visser 1996 and Nahin 1999). When a space-time is such that it admits chronology violating regions there seems that information can be generated from nothing. Lewis (1976) has already written on casual loops involving just information transfer. He concluded that there is simply no answer to the question on the origin of the information, and that casual loops of this kind are not too different from other situations we are confronted with, such as the origin of the universe or the decay of a tritium atom. A similar view is sustained by Levin (1980), who compares casual loop paradoxes to questions about where anything originally comes from. CTCs can confront us with a way of producing knowledge that conflicts with the principles of philosophy of science, since knowledge can come into existence without any evolutionary process (Deutsch 1991). However, it is important to notice that information paradoxes are not paradoxes at all, in the sense that they do not imply any contradiction with logical or even physical laws. They just seem to contradict our common sense, but this is not enough to rule them out.

In an recent twist of the problem, Smith (1997) argues that a comparable example of information coming from nothing could be described as follows. Suppose that someone says something trivial, but other person mishears him, thinking that he has said something profound. Smith asks, "where does this profound idea come from?". It seems to have been "spontaneously" generated and, we are told, this would be similar to what happens with information in a CTC. We sustain, on the contrary, that both situations are completely different. The formation of ideas and concepts is a brain process. We say that an idea has emerged when certain neural plastic networks are activated. The activation mechanism, although not clear, is surely far from linear. Small changes in the input signals can trigger quite different responses. In particular, a small perturbation in the process of hearing a speech can result in a different chain of thoughts in the listener (see Bunge 1980 and references therein for more details on the ideation process). It is clear then that in Smith's situation, the idea comes from electro-chemical processes in a brain and not from nowhere. Even if we consider the information "carried" by the idea, this information does not come from nowhere; it is produced in the brain of a human being. Furthermore, Smith's method of producing information is very limited: it can not be used to transmit an information volume comparable to a whole book, but just some phrases, probably ultimately related to the course of a previous conversation. The phenomenon quoted by Smith is, besides, local in time, whereas information produced by CTCs is not. 
In casual loops, information simply exists as a consequence of the peculiar topology of the space-time manifold.

\subsection{Information loops and the PSC}

Consider the local light cone of a time traveler. There are three, and only three, possible final destinations for a time trip. No matter the way in which the travel is done, the final destiny of the traveler can only be one out of three possibilities: either a she arrives inside her past light cone, or $\mathbf{b}$ she arrives on the light cone, i.e. at a distance equal (in geometrized units) to the time that light would take to come back to the initial position through normal space, or $\mathbf{c}$ she arrives at a point out of her initial light cone. For the first two possibilities, an information loop is obviously unavoidable. For the third one, when the traveler arrives at a point out of her initial past light cone, there is no information loop with only one time trip. Traveling at the speed of light, information sent back to the initial position of the time machine will always arrive after the time trip commenced. But even in the last case, if more than one time machine are available (for instance as in the situation known as a Roman ring (Visser 1997), where there are two wormholes in relative motion) the past might be affected by information flux from the future. However, with just one time machine, affecting the past is impossible in case c. Advocates of chronology protection could then invoke that just this kind of time travel is possible in nature. This requirement could be stated as a Conjecture of Past Shielding: every single-time-machine time trip would be such that the two extreme points are always separated by a space-like interval. In order to enforce this conjecture it could be pointed out that, if natural wormholes exist at all, their number would be so scarce that a Roman ring configuration (Visser 1997) would well be practically impossible (Torres 1998a, b; Anchordoqui 1999, see -on the same topic- Eiroa et al. 2000 and Safonova et al. 2000a, b).

However, the conclusion is that whatever the final destiny of the time-traveler is, in principle, she could always affect her own past. Otherwise stated, chronology-violating space-times generally admit information loops: they cannot be excluded on the only basis of the PSC.

Although the PSC do not preclude that within chronology-violating regions information steaming from the future can affect the past, it at least imposes constraints on the way this can be done. In fact, any physical process causally triggered by the backwards information flux must be consistent with the past history of the universe. This means that if a time traveler goes back to his past and tries, for instance, to communicate the contents of the theory of special relativity to the scientific community before 1905, she will fail because at her departure it was historically clear that the first paper on special relativity was published by Albert Einstein in June 1905. The details of her failure will depend on the details of her travel and attempt, in the same way that the details of the failure of a perpetual motion machine depends on the approach used by the imprudent inventor. All we can say a priori is that the laws of physics are such that these attempts cannot succeed and information cannot propagate arbitrarily in space-time. It is precisely due to the PSC that we know that our past is not significantly affected from the future: we know that till now, knowledge has been generated by evolutionary processes, i.e. there is small room in history for information loops. This does not necessarily mean that the same is valid for the entire space-time.

\section{Conclusions}

The field equations of GTR, as well as those of other classical theories of gravity, have solutions that allow the formation of closed time-like curves. Some of these solutions occur under conditions that could happen in the real universe, at least as far as our present knowledge let us see. Although a full theory of quantum gravity could, perhaps, provide some effective mechanism to enforce chronology protection, the success of 
the classical theory over a very wide range of applications makes commendable the study of the expected behaviour of physical systems in the presence of CTCs. As remarked by Thorne (1992), through pushing the theory to its most extreme predictions, we can get important insights of its limitations and, hopefully, the ways to supersede them. From this point of view, time travel is more than a mere justification for theoretical speculation; it is a conceptual tool that can be used to probe the deepest levels of General Relativity and the picture of the world that emerges from this theory; it is an epistemological instrument that can lead us to an important clarification of the very foundations of space-time theories and their implications.

In this paper we have shown that the Principle of Self-Consistency excludes the possibility of a bizarre ontology with self-existing objects. We also have suggested that this principle is a metanomological statement that enforces the global-to-local harmony in the network of laws that determine the evolution of physical systems in space-time. The information flux from future to the past is possible in GTR, although restricted by consistency constraints. This means that past and future are not longer globally distinct when CTCs occur and that causality can globally -although never locally- operate backwards.

\section{Acknowledgments}

This work has been supported by the agencies CONICET and ANPCT (PICT 03-04881) as well as by funds granted by Fundación Antorchas to both authors.

\section{References}

[1] Anchordoqui, L. A., Grunfeld, A. G. and Torres, D. F. (1998): 'Vacuum Static Brans-Dicke Wormhole', Gravitation and Cosmology, 4, pp. 287-290.

[2] Anchordoqui, L. A., Perez Bergliaffa, S. E. and Torres, D. F (1997): 'Brans-Dicke Wormholes in Non-Vacuum Space-Times', Physical Review, D55, pp. 5226-5229.

[3] Anchordoqui, L. A., Romero, G. E., Torres, D. F. and Andruchow, I. (1999): 'In Search for Natural Wormholes', Modern Physics Letters, A14, pp. 791-798.

[4] Bunge, M. (1961): 'Laws of Physical Laws', American Journal of Physics, 29, pp. 518-529.

[5] Bunge, M. (1980): The Mind-Body Problem, Pergamon, Oxford.

[6] Carlini, A., et al. (1995): 'Time Machines: The Principle of Self-Consistency as a Consequence of the Principle of Minimal Action', International Journal of Modern Physics, D 4, pp. 557-580.

[7] Cassidy, M. J. and Hawking, S. W. (1998): 'Models for Chronology Selection', Physical Review, D57, pp. 2372-2380.

[8] Deutsch, D. (1991): 'Quantum Mechanics Near Closed Time-Like Lines', Physical Review, D44, pp. 3197-3217.

[9] Earman, J. (1995a): Bangs, Crunches, Whimpers, and Shrieks: Singularities and Acausalities in Relativistic Spacetimes, Oxford University Press, New York.

[10] Eiroa, E., Romero, G. and Torres D. F. (2000): 'Chromaticity effects in microlensing by wormholes' Modern Physics Letters A16, pp. 973-983. 
[11] Earman, J. (1995b): 'Outlawing Time Machines: Chronology Protection Theorems', Erkenntnis, 42, pp. 125-139.

[12] Friedman, J.L., et al. (1990): 'Cauchy Problem in Spacetimes with Closed Timelike Curves', Physical Review, D42, pp. 1915-1930.

[13] Frolov, V. and Novikov, I. D. (1990): 'Physical Effects in Wormholes and Time Machines', Physical Review, D42, pp. 1057-1065 .

[14] Godfrey-Smith, W. (1980): 'Travelling in Time', Analysis, 40, pp. 72-73.

[15] Gödel, K. (1949): 'An Example of a New Type of Cosmological Solution of Einsteins Field Equations of Gravitation', Reviews of Modern Physics, 21, pp. 447-450.

[16] Harrison, J. (1979): 'Report on Analysis Problem No. 18', Analysis, 39, pp. 65-69.

[17] Hawking, S. W. (1992): 'Chronology Protection Conjecture', Physical Review, D46, pp. 603-611.

[18] Hochberg, D. and Visser, M. (1997): 'Geometric structure of the generic wormhole throat', Physical Review, D56, pp. 4745-4755.

[19] Kim, S. -W. and Thorne, K. S. (1991): 'Do Vacuum Fluctuations Prevent the Creation of Closed Timelike Curves?', Physical Review, D43, pp. 3929-3947.

[20] Konstantinov, M. Y. (1995): 'Comments on "The Principle of Self-Consistency as a Consequence of

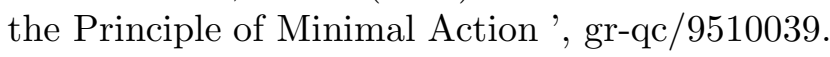

[21] Landau, L. D. and Lifchitz, E. M. (1975): Fluid Mechanics, Pergamon, Oxford.

[22] Levin, M. R. (1980): 'Swords' Point', Analysis, 40, pp. 69-70.

[23] Lewis, D. (1976): 'Paradoxes of Time Travel', American Philosophical Quaterly, 13, pp. 145-152.

[24] Li, L., -X. (1994): 'New Light on Time Machines: Against the Chronology Protection Conjecture', Physical Review, D50, pp. R6037-R6040.

[25] Li, L. -X., Xu, J. -M, Liu, L. (1993): 'Complexd Geometry, Quantum Tunneling, and Time Machines', Physical Review, D48, pp. 4735-4737.

[26] MacBeath, M. (1982): 'Who was Dr. Who's father', Synthese, 51, pp. 397-430.

[27] Misner, C. W., Thorne, K. S. and Wheeler, J. A. (1973): Gravitation, W. H. Freeman and Co., San Francisco.

[28] Morris, M. S. and Thorne, K. S. (1988): 'Wormholes and Their Use for Interstellar Travel: A Tool for Teaching General Relativity', American Journal of Physics, 56, pp. 395-412.

[29] Morris, M. S., Thorne, K. S. and Yurtsever, U. (1988): 'Wormholes, Time Machines and the Weak Energy Condition', Physical Review Letters, 61, pp. 1446-1449.

[30] Nahin, P. J. (1999): Time Machines: Time Travel in Physics, Metaphysics and Science Fiction, Springer-Verlag and AIP Press, New York.

[31] Nerlich, G. (1981): 'Can Time Be Finite?', Pacific Philosophical Quaterly, 62, pp. 227-239. 
[32] Safonova, M., Torres, D. F. and Romero G. E. (2000a): 'Macrolensing signatures of large-scale violations of the weak energy condition', Modern Physics Letters A16, pp. 153-162.

[33] Safonova, M., Torres, D. F. and Romero G. E. (2000b): 'Microlensing by natural wormholes: theory and simulations', [igr-qc/0 105070$]$, Submitted.

[34] Smith, N. J. J. (1997): 'Bananas Enough for Time Travel?', British Journal for the Philosophy of Science, 48, pp. 363-389.

[35] Thorne, K.S. (1992): 'Closed Timelike Curves', in General Relativity and Gravitation 1992, Gleiser, J. L., et al. eds., Institute of Physics, Bristol.

[36] Torres, D. F., Romero, G. E. and Anchordoqui, L. A. (1998a): 'Might Some Gamma Ray Bursts Be the Observable Signature of Natural Wormholes?', Physical Review, D58, 123001.

[37] Torres, D. F., Romero, G. E. and Anchordoqui, L. A. (1998b): 'Womholes, Gamma Ray Bursts and the Amount of Negative Mass in the Universe', Modern Physics Letters, A13, pp. 1575-1582.

[38] Visser, M. (1996): Lorentzian Wormholes, AIP Press, New York.

[39] Visser, M (1997): 'The Roman Ring', Physical Review, D55, pp. 5212-5214. 